\title{
Effectiveness of early treatment of lopinavir-ritonavir in patients with severe COVID-19: a case series
}

\author{
Authors: Pan Luo, ${ }^{\mathrm{A}}$ Jian-ling Zheng, ${ }^{\mathrm{A}}{ }^{*}$ Yi Liu, ${ }^{\mathrm{B}} \mathrm{Lin}$ Qiu, ${ }^{\mathrm{A}}$ Xiu-lan Liu, ${ }^{\mathrm{A}}$ Hui-ying Xue, ${ }^{\mathrm{A}}$ Dong $\mathrm{Liu}^{\mathrm{C}+}$ and Juan $\mathrm{Li}^{\mathrm{C}+}$
}

\section{Aim}

The inconsistent effects of lopinavir-ritonavir (LPV/r) on COVID-19 seem to be caused by the therapeutic window. In the present study, we aim to present the effects of early LPV/r treatment on patients with severe COVID-19.

\section{Methods}

The demographics, characteristics, treatments, SARS-CoV-2 test results and outcomes of 19 patients with severe COVID-19 treated with LPV/r within 12 days of onset of symptoms were retrospectively assessed.

\section{Results}

Within 3 days of admission, three (15.79\%) patients received noninvasive ventilation, and 16 (84.21\%) patients received highflow oxygen support. The median duration between the onset of symptoms and initiating LPV/r therapy was 9 (range 2-12) days. The median course of LPV/r treatment was 11 (range 7-17) days. One of the 19 patients $(5.26 \%)$ died. Of the 18 patients discharged, the median hospital stay was 17 (range 11-45) days. At day 6 after LPV/r therapy was initiated, $68.42 \%$ of patients were virologically cured, increasing to $84.22 \%$ at day 12 .

\section{Conclusion}

In this cohort of patients with severe COVID-19 who were treated with LPV/r within 12 days of the onset of symptoms, clinical improvement was observed in $18 / 19$ patients $(94.74 \%)$. Randomised controlled trials are urgently needed to further evaluate this strategy.

KEYWORDS: lopinavir-ritonavir, SARS-CoV-2, COVID-19

DOI: $10.7861 /$ clinmed.2020-0348

\section{Introduction}

One of the most promising drugs for the treatment of coronavirus disease 2019 (COVID-19) is lopinavir-ritonavir (LPV/r). But it is

Authors: Aresponsible pharmacist, Tongji Hospital, Tongji Medical College, Huazhong University of Science and Technology, Wuhan, China; ${ }^{B}$ associate chief pharmacist, Tongji Hospital, Tongji Medical College, Huazhong University of Science and Technology, Wuhan, China; 'Chief pharmacist, Tongji Hospital, Tongji Medical College, Huazhong University of Science and Technology, Wuhan, China; *joint first authors; ${ }^{+}$joint corresponding authors dispiriting that no benefit has been observed with LPV/r treatment in recent open-label randomised controlled trials in adult patients hospitalised with COVID-19.,2 However, in a posthoc subgroup analysis of the patients who were treated within 12 days after the onset of symptoms, the mortality in standard-care group was numerically greater than the LPV/r group.' It seems that early intervention with LPV/r may be of benefit in COVID-19. This hypothesis was also supported by a retrospective analysis which identified that COVID-19 patients may benefit from sustained lopinavir-combined regimen with a median length of time between the onset of symptoms and therapy initiation of 5 days (interquartile range [IQR] 3-6). ${ }^{3}$ At present, the results regarding the efficacy of LPV/r treatment in COVID-19 show inconsistency between studies. ${ }^{4-7}$ Whether this is caused by the therapeutic window of LPV/r is still not well illustrated. In this retrospective observational study, we aim to present the effects of an early intervention with LPV/r on patients with severe COVID-19 in a single centre, providing additional evidence on the clinical use of LPV/r in COVID-19.

\section{Methods}

\section{Study design and participants}

This retrospective study included patients with confirmed COVID-19 who were treated with LPV/r and discharged or died from 27 January 2020 to 24 March 2020 at Tongji Hospital in Wuhan, China. The inclusion criteria of our study were patients aged 18-80 years with molecularly proven SARS-CoV-2 infections who were classified as severe cases on admission and who received LPV/r treatment as least 3 days. Cases where the length of time between the onset of symptoms and initiating LPV/r therapy was $>12$ days, where LPV/r therapy was used in combination with other antiviral(s) after admission, or where there was insufficient information of the evaluation indexes were excluded from the study. The criteria for defining severe COVID-19 were derived from the literature..$^{8,9}$ The data, including demographics, clinical characteristics, treatments, results of oropharyngeal and nasopharyngeal swab tests for SARS-CoV-2 nucleic acid, and patient outcomes (mortality and the hospitalisation time) were obtained from the medical records. The study was approved by the ethical committee of Huazhong University of Science and Technology.

\section{Statistics}

Data are presented as median (range) or as the number and percentage, as appropriate. 
Table 1. Clinical characteristics and outcomes of severe COVID-19 patients treated with lopinavir-ritonavir (LPV/r) within 12 days of onset of symptoms

\begin{tabular}{|c|c|c|c|c|c|c|c|c|}
\hline $\begin{array}{l}\text { Case } \\
\text { No. }\end{array}$ & Sex & Age & $\begin{array}{l}\text { Category of } \\
\text { oxygen support }\end{array}$ & Comorbidity & $\begin{array}{l}\text { Symptom onset } \\
\text { to LPV/r therapy } \\
\text { (days) }\end{array}$ & $\begin{array}{l}\text { Course of LPV/r } \\
\text { use (days) }\end{array}$ & $\begin{array}{l}\text { Clinical } \\
\text { outcomes }\end{array}$ & $\begin{array}{l}\text { Hospitalisation } \\
\text { time (days) }\end{array}$ \\
\hline 1 & M & 63 & $\begin{array}{l}\text { Non-invasive } \\
\text { ventilation }\end{array}$ & None & 9 & 12 & Discharge & 44 \\
\hline 2 & M & 32 & High-flow oxygen & $\begin{array}{l}\text { Hepatitis B } \\
\text { infection }\end{array}$ & 9 & 7 & Discharge & 17 \\
\hline 3 & M & 71 & $\begin{array}{l}\text { Non-invasive } \\
\text { ventilation }\end{array}$ & None & 11 & 8 & Death & N/A \\
\hline 4 & M & 73 & High-flow oxygen & $\begin{array}{l}\text { Rheumatoid } \\
\text { arthritis }\end{array}$ & 4 & 10 & Discharge & 15 \\
\hline 5 & M & 55 & High-flow oxygen & $\begin{array}{l}\text { Hypertension } \\
\text { Haemorrhage }\end{array}$ & 7 & 12 & Discharge & 42 \\
\hline 6 & M & 67 & High-flow oxygen & None & 6 & 10 & Discharge & 13 \\
\hline 7 & M & 57 & High-flow oxygen & None & 10 & 7 & Discharge & 45 \\
\hline 8 & M & 77 & High-flow oxygen & Gout & 12 & 12 & Discharge & 29 \\
\hline 9 & $\mathrm{~F}$ & 75 & High-flow oxygen & None & 8 & 14 & Discharge & 22 \\
\hline 10 & $\mathrm{~F}$ & 57 & $\begin{array}{l}\text { Non-invasive } \\
\text { ventilation }\end{array}$ & $\begin{array}{l}\text { Hypertension, } \\
\text { diabetes }\end{array}$ & 11 & 11 & Discharge & 39 \\
\hline 11 & M & 46 & High-flow oxygen & Hypertension & 12 & 10 & Discharge & 11 \\
\hline 12 & $\mathrm{~F}$ & 65 & High-flow oxygen & None & 3 & 12 & Discharge & 17 \\
\hline 13 & M & 33 & High-flow oxygen & None & 11 & 15 & Discharge & 15 \\
\hline 14 & $\mathrm{~F}$ & 46 & High-flow oxygen & None & 5 & 14 & Discharge & 17 \\
\hline 15 & M & 61 & High-flow oxygen & Diabetes & 11 & 9 & Discharge & 14 \\
\hline 16 & $\mathrm{~F}$ & 38 & High-flow oxygen & None & 2 & 11 & Discharge & 13 \\
\hline 17 & $\mathrm{~F}$ & 57 & High-flow oxygen & None & 5 & 17 & Discharge & 35 \\
\hline 18 & $\mathrm{~F}$ & 72 & High-flow oxygen & None & 12 & 10 & Discharge & 27 \\
\hline 19 & M & 42 & High-flow oxygen & Diabetes & 9 & 12 & Discharge & 17 \\
\hline
\end{tabular}

\section{Results}

19 patients (12 males and seven females) with severe COVID-19 were included in this study. They were treated with LPV/r (400 mg and $100 \mathrm{mg}$ ) twice daily on the day of admission or on the day after. Patients received symptomatic treatment, antibiotics and supportive therapies as appropriate. The characteristics, treatment, and outcomes of the patients are summarised in Table 1. The median age of the patients was 57 (range 32-77) years. Eight (42.11\%) patients had one or more comorbidities at admission, including hepatitis B infection, rheumatoid arthritis, hypertension, hemorrhage and diabetes. Within 3 days of admission, three (15.79\%) patients received noninvasive ventilation, and $16(84.21 \%)$ received high-flow oxygen support. The median duration between the onset of symptom and initiating LPV/r therapy was 9 (range 2-12) days. The median course of LPV/r use was 11 (range 7-17) days. One of the 19 patients (5.26\%) died, and the 18 remaining patients (94.74\%) have recovered and been discharged. Of the 18 patients who were discharged, the median hospital stay was 17 (range 11-45) days.
A rapid fall in the viral-positive rate in oropharyngeal and nasopharyngeal swabs tested by PCR was noted (Fig 1). At day 6 from initiation of the LPV/r therapy, $68.42 \%$ of patients were virologically cured, and $84.22 \%$ of the patients were virologically cured at day 12 .

\section{Discussion}

This retrospective report describes the clinical outcomes in a small cohort of COVID-19 patients who were treated with LPV/r no more than 12 days after the first onset of symptoms. Specifically, the in-hospital mortality was $5.26 \%$, and $94.74 \%$ of patients have recovered and been discharged. The median hospital stay was 17 (range $11-45$ ) days, and $68.42 \%$ of patients were virologically cured at day 6 from LPV/r therapy initiation and $84.22 \%$ of patients cured at day 12 .

In a recent randomised controlled trial of LPV/r in adults hospitalised with severe COVID-19 conducted by Cao et al, the 28-day mortalities of the standard-care group treated within 12 days after the onset of symptom was $27.1 \%$. 'By way of comparison, the $5.26 \%$ mortality observed in this LPV/r use cohort is noteworthy 


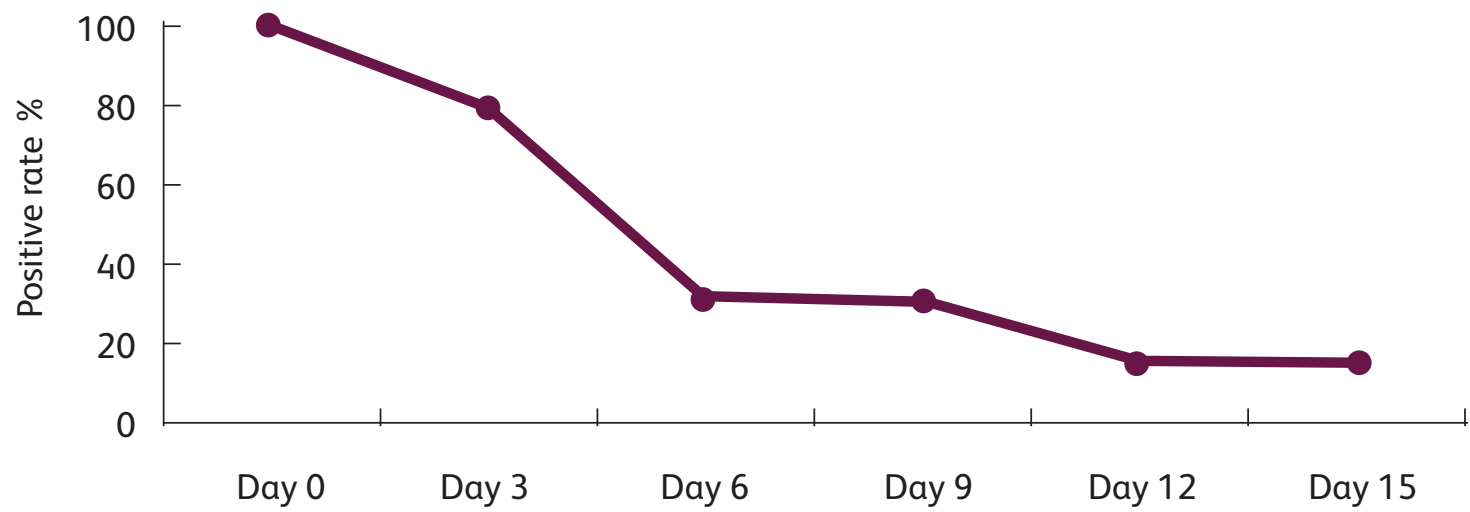

Fig 1. The change of SARS-CoV-2 positive rate over time. The fall in culture positivity from the third day after the onset of LPV/r therapy is remarkable. At day 6 after LPV/r therapy was initiated, $68.42 \%$ of patients were virologically cured and $84.22 \%$ of patients were virologically cured at day 12 .

since the age, oxygen-support category, and comorbidities between this LPV/r use cohort and the standard-care group of Cao et al's trial were comparable at admission. Moreover, in a cohort of patients hospitalised for severe COVID-19 who were treated with remdesivir, the mortality in patients receiving noninvasive oxygen support was $5.3 \% .^{10}$ This mortality rate is very close to our results of $5.26 \%$. It is important to note that it was suggested that the use of remdesivir in patients with severe COVID-19 may have clinical benefit.10

Patients with clinical improvement and two consecutive negative PCR tests can be discharged according to national guidelines. However, a stricter policy followed in some hospitals, including ours, is that several days of observation is also required before COVID-19 patients are discharged. Therefore the length of stay in this cohort seems not to be directly comparable to those in other reports. Despite this, the hospital stays (median 17 days, range $11-45$ days) of the discharged patients in this LPV/r use cohort is still encouraging, since Liu et a ${ }^{11}$ reported a median length of stay of 25 (range 14-44) days, Zheng et al ${ }^{12}$ a median length of stay of 35.97 days and Zhou et $a l^{13}$ a median time from illness onset to discharge of 40.0 (IQR 32.0-46.0) in severe COVID-19 patients.

The favourable evolution of our patients under an early LPV/r treatment paralleled a relatively rapid negative test for SARSCoV-2. These data are important to compare with those in the literature, which shows that the median duration of viral shedding was 31 (IQR: 24-40) days from illness onset. ${ }^{13}$ There was also research reported that the number of days required for $\mathrm{nCoV}$-RNA turning negative was $12.0 \pm 0.82$ in patients who were just treated with adjuvant medicine. ${ }^{5}$ However, the fall in culture positivity from the third day after the onset of LPV/r therapy is remarkable in our cohort and is one element suggesting the effectiveness of this treatment. A recent study showed that patients who initiated LPV/r treatment within 10 days from symptom onset, but not those who initiated treatment from day 11 onwards, had significantly shorter viral shedding duration compared with those without LPV/r treatment, ${ }^{14}$ which was consistent with our findings.

The results from the recently randomised evaluation of COVID-19 therapy (RECOVERY) trial showed that LPV/r was not associated with reductions in 28-day mortality, duration of hospital stay, or risk of progressing to invasive mechanical ventilation or death. ${ }^{2}$ This finding was contrast with our results. However, RECOVERY included patients who did not initiate
$\mathrm{LPV} / \mathrm{r}$ therapy until $>12$ days after the onset of symptoms. Therefore, the therapeutic window of LPV/r treatment could be an explanation for the contradictions. Direct acting antiviral drugs are usually more effective when used earlier during the disease course, corresponding to reports from the literature that early administration of LPV/r could prevent COVID-19.,34-16 Moreover, the course of treatment, clinical severity, the comorbidities of patients and the adjuvant drugs used may also influence the effects of LPV/r in different studies.

In this retrospective study, data on the safety and side-effects of $\mathrm{LPV} / \mathrm{r}$ were not collected because attribution of the dysfunction to either LPV/r or the COVID-19 is challenging. Interpretation of the results in this descriptive pilot study is limited by the small size of the cohort, and we did not attempt an analytic approach to account for possible confounds. Nor did we evaluate the clinical relevance of the patients' co-morbidities on the effects of LPV/r or he potential risk of drug-drug interactions between LPV/r and comedications used for the management of other comorbidities in these patients. But for all this, the preliminary data in this cohort suggest that early intervention with LPV/r treatment in patients with severe COVID-19 could be effective and randomised controlled trials are urgently needed to further evaluate this strategy.

\section{References}

1 Cao B, Wang Y, Wen D et al. A trial of lopinavir-ritonavir in adults hospitalized with severe COVID-19. N Engl J Med 2020;382: 1787-99.

2 RECOVERY Collaborative Group. Lopinavir-ritonavir in patients admitted to hospital with COVID-19 (RECOVERY): a randomised, controlled, open-label, platform trial. Lancet 2020;396:1345-52.

3 Liu F, Xu A, Zhang Y et al. Patients of COVID-19 may benefit from sustained lopinavir-combined regimen and the increase of eosinophil may predict the outcome of COVID-19 progression. Int J Infect Dis 2020;95:183-91.

4 Zhu Z, Lu Z, Xu T et al. Arbidol monotherapy is superior to lopinavir/ ritonavir in treating COVID-19. J Infect 2020;81:e21-e3.

5 Ye XT, Luo YL, Xia SC et al. Clinical efficacy of lopinavir/ritonavir in the treatment of Coronavirus disease 2019. Eur Rev Med Pharmacol Sci 2020;24:3390-6.

6 Panagopoulos P, Petrakis V, Panopoulou M et al. Lopinavir/ ritonavir as a third agent in the antiviral regimen for SARS-CoV-2 
infection. J Chemother 2020, in press (doi: 10.1080/1120009X. 2020.1775424).

7 Hariyanto TI, Kristine E, Jillian Hardi C, Kurniawan A. Efficacy of lopinavir/ritonavir compared with standard care for treatment of coronavirus disease 2019 (COVID-19): a systematic review. Infect Disord Drug Targets 2020, in press (doi: 10.2174/1871526520666 201029125725)

8 Huang I, Lim MA, Pranata R. Diabetes mellitus is associated with increased mortality and severity of disease in COVID-19 pneumonia - A systematic review, meta-analysis, and metaregression. Diabetes Metab Syndr 2020;14:395-403.

9 Luo P, Qiu L, Liu Y et al. Metformin treatment was associated with decreased mortality in COVID-19 patients with diabetes in a retrospective analysis. Am J Trop Med Hyg 2020;103:69-72.

10 Grein J, Ohmagari N, Shin D et al. Compassionate use of remdesivir for patients with severe Covid-19. N Engl J Med 2020;382: 2327-36.

11 Liu X, Zhou H, Zhou Y et al. Risk factors associated with disease severity and length of hospital stay in COVID-19 patients. J Infect 2020;81:e95-e97.

12 Zheng $\mathrm{Y}, \mathrm{Xu} \mathrm{H}$, Yang $\mathrm{M}$ et al. Epidemiological characteristics and clinical features of 32 critical and 67 noncritical cases of COVID-19 in Chengdu. J Clin Virol 2020;127:104366.
13 Zhou B, She J, Wang Y, Ma X. The duration of viral shedding of discharged patients with severe COVID-19. Clin Infect Dis 2020;71:2240-2.

14 Yan D, Liu XY, Zhu YN et al. Factors associated with prolonged viral shedding and impact of lopinavir/ritonavir treatment in hospitalised non-critically ill patients with SARS-CoV-2 infection. Eur Respir ] 2020;56:2000799.

15 Klement-Frutos E, Burrel S, Peytavin G et al. Early administration of ritonavir-boosted lopinavir could prevent severe COVID-19. J Infect 2020, in press (doi: 10.1016/j.jinf.2020.05.039).

$16 \mathrm{Kim}$ JW, Kim EJ, Kwon $\mathrm{HH}$ et al. Lopinavir-ritonavir versus hydroxychloroquine for viral clearance and clinical improvement in patients with mild to moderate coronavirus disease 2019. Korean ] Intern Med 2020 (doi: 10.3904/kjim.2020.224).

Address for correspondence: Prof Juan Li, Department of Pharmacy, Tongji Hospital, Tongji Medical College, Huazhong University of Science and Technology, Wuhan 430030, China. E-mail: lijuan@tjh.tjmu.edu.cn 